\section{RT-PCR method for selective detection of silent gene transcripts in silencing mutants in homothallic strains of Schizosaccharomyces pombe}

\author{
Shakil Ahmed and Jagmohan Singh \\ Institute of Microbial Technology, Chandigarh, India
}

BioTechniques 44:54-58 (January 2008)

doi $10.2144 / 000112671$

Here we describe a method that allows selective detection of silent copy transcripts in homothallic strains of Schizosaccharomyces pombe in the presence of the active cassettes. The method involving RT-PCR (reverse transcriptase polymerase chain reaction) exploits our observation that the silent copy transcripts extend beyond the regions of homology to the flanking sequences specific for the donor cassettes, thus allowing design of oligos that are specific for the different donors. The results are validated using a known silencing mutant swi6.
With the emergence of Schizosaccharomyces pombe as a model system for gene silencing (1), there is a need for simpler and faster methods for detection of silent copy transcripts. The common biochemical approach for detecting the silent gene transcripts in the silencing mutants of $S$. pombe involves Northern hybridization, wherein a strain with stable $M$ mating type is used and the transcripts from the mat $2 P$ locus are detected by probing the Northern blots with plus (+) allelespecific probe (2). Alternatively, the RT-PCR (reverse transcription polymerase chain reaction) approach was used to amplify either mat $2 P$ or mat $3 M$ specific transcripts in a strain carrying a deletion of the mat 1 locus (3). However, detection of silent gene transcripts in silencing mutants with a homothallic genetic background by the abovementioned approaches is not feasible because of contribution of similar plus (+) and minus (-) allele-specific products by the mat 1 locus as well.

We have arrived at a solution to this problem on the basis of our finding that the mat $2 P$ - (and possibly mat $3 M-)$ specific transcripts extend beyond the homology regions $H 2$ and $H 3$ (Figure 1). It has been shown earlier that the matl-specific transcripts matlPc and matlMi both extend beyond the $H 2$ region of mat 1 in the centromere-proximal direction, yielding 750 and 800 nucleotide-long transcripts, respectively (4). We asked the question whether the transcripts originating from the mat $2 P$ (mat $2 P c$ transcript) and mat $3 M$ (mat $3 \mathrm{Mi})$ also extend beyond the homology region $\mathrm{H3}$, which is common to both these cassettes. To test this, an oligo (dT) primer was used to make the cDNA copy of the total RNA and the $\left[\gamma^{32} \mathrm{P}\right]-$ labeled plus or minus allele-specific oligos were used to PCR amplify the $P c$ and $M i$ transcripts both from the expressed matl locus and the silent locus mat $2 P$. We found that in addition to the $750 \mathrm{bp}$ product specific for matlPc (4), another band of about $800 \mathrm{bp}$ was also observed in the silencing mutants, which presumably originated from the donor locus mat $2 P$ and extended about $300 \mathrm{bp}$ outside the $\mathrm{H} 3$ region (data summarized in Figure 1). Although a similar mapping was not done for mat $3 M$, the results shown below indicate that $M i$ transcript from mat $3 M$ also extends well past the $H 3$ box. From these data, we infer that the transcript $P c$ transcribed from mat $2 P$, and possibly the transcript $M i$ transcribed from mat $3 M$ (this prediction was corroborated by the experiments below), extend far outside not only the $H 2$ box but the $H 3$ box as well. Therefore, without having to map the $3^{\prime}$ ends of the donor-specific transcripts, it should be possible to amplify the mat $2 P c$ and $m a t 3 M i$ transcripts specifically by using the oligos located immediately outside the $H 3$ box in the mat $2 P$ and mat $3 M$ loci, respectively. It has been shown that $P c$ transcript is constitutively expressed but stimulated further
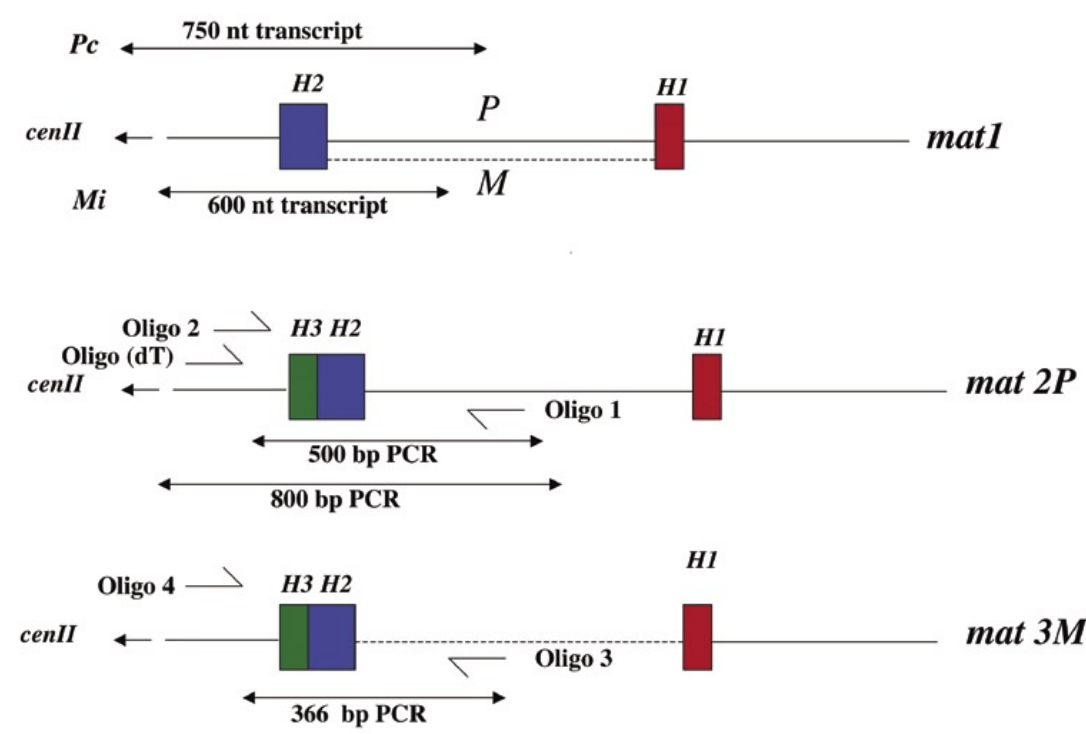

Figure 1. Schematic representation of the mating type loci mat1P/M, mat2P, and mat3M. The lengths of the $P c$ and $M i$ transcripts from $m a t l$ and PCR products from $m a t 2 P$ and $m a t 3 M$ are indicated. The relative location of the oligos within and outside the donor cassettes used for PCR and the predicted sizes of the PCR fragments ( $500 \mathrm{bp}$ for $m a t 2 P c$ and $366 \mathrm{bp}$ for $m a t 3 \mathrm{Mi}$ ) are indicated. 


\section{Benchmarks}

by nitrogen starvation (4). On the other hand, the $M i$ transcript is not expressed at all in the nitrogen-rich medium and is induced upon nitrogen starvation (4). Further, it has been shown that the silent loci are also derepressed in the swi6 mutant (5). Therefore, we chose swi6 mutant as a representative silencing mutant.

Suitable antisense oligos were designed that are located immediately proximal to the $H 3$ region in the donor loci (Table 1). The whole procedure was carried out in the following steps:

1. Total RNA was isolated from homothallic $\left(h^{90}\right)$ wild-type and swi6 mutant strains grown in nitrogen-rich and nitrogen-free medium (6) by the hot phenol method (7).

2. cDNA synthesis was carried out with 10-15 $\mu \mathrm{g}$ RNA using the oligo (dT) primer (Table 1) with MuLV reverse transcriptase (New England Biolabs, Ipswich, MA, USA), using the manufacturer's protocol.

3. The cDNA product was diluted 10-fold and PCR reactions were carried out in $50 \mu \mathrm{l}$, using the primer pair 1 and 2 for $m a t 2 P c$ and primer pair 3 and 4 for mat $3 M i$ (Table 1). The PCR conditions were: $94^{\circ} \mathrm{C}, 1 \mathrm{~min} ; 55^{\circ} \mathrm{C}, 1 \mathrm{~min}$; and $72^{\circ} \mathrm{C}, 2 \mathrm{~min}$; $15-20$ cycles. Under these conditions, we have found that we can compare the quantities of the RNA levels as this condition occurs during the logarithmic phase of the amplification. The PCR products were resolved by agarose gel electrophoresis, followed by Southern blotting (8). Hybridization was carried out with the random primer labeled probes (9) prepared from the plasmids containing the mat $2 P$ and $m a t 3 M$ fragments. The protocol is outlined in Table 2.

Results are shown in Figure 2. An interesting observation is the presence of the PCR product of 500 bp specific for mat $2 P c$ transcript, at a low level in nitrogen-rich medium, which is significantly elevated under nitrogen starvation conditions in wild-type samples (Figure 2, lane 2).

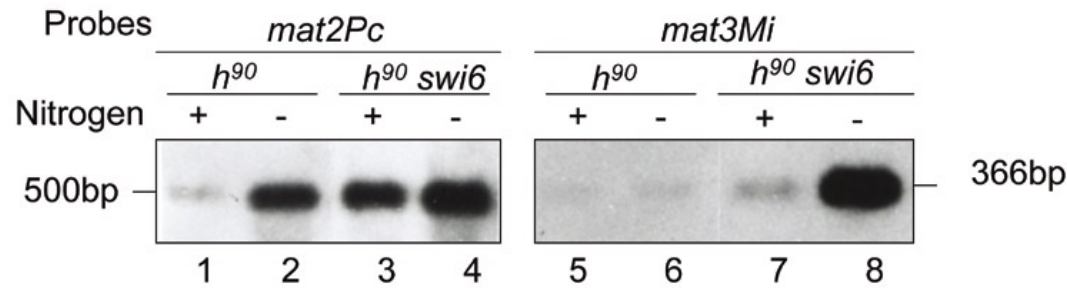

Figure 2. RT-PCR analysis of the mat2Pc and mat3Mi transcripts in wild-type, $h^{y 0}$ (homothallic) and $\boldsymbol{h}^{90}$, swi6 strains. RNA was isolated from wild-type (lanes $\left.1,2,5,6\right)$ and swi6 $(3,4,7,8)$ mutant cells grown in nitrogen-rich medium (lanes $1,3,5,7$; indicated by + sign) and nitrogen-free medium (lanes 2, 4, 6, 8; indicated by - sign), and cDNA synthesis and mat $2 P c$ - and mat $3 M i$-specific PCR reactions were carried out as described earlier $(4,7)$. The PCR products were resolved by agarose gel electrophoresis, subjected to Southern blotting and hybridization as described (8). The probes used were prepared from the plasmid DNAs of mat $2 P$ and $m a t 3 M$ by PCR using the same oligo pairs; oligos 1 and 2 for $m a t 2 P c$ and oligos 3 and 4 for $m a t 3 M i$.

Table 1. List of the Oligos Used for cDNA Synthesis and PCR Reaction

\begin{tabular}{|ll|}
\hline Name of the Oligo & $\mathbf{5}^{\prime}$ - Sequence of the oligo $\mathbf{- 3}{ }^{\prime}$ \\
\hline Oligo (dT) primer & AATTCTGCAGTGATATCTTTTTTTTTTTTTTTTT \\
Oligo 1 (mat2Pc sense) & GATTAAGAGCACCTATTTTCTTGCC \\
Oligo 2 (mat2Pc antisense) & CAACGGATTACTAAAAACAGTTTAAATG \\
Oligo 3 (mat3Mi sense) & CATACTAATAATGTCAGCAGAAGAC \\
Oligo 4 (mat3Mi antisense) & CCAATCAACTTAACATGAAGCAACTCCTGATAC \\
\hline
\end{tabular}

Interestingly, a high level of mat $2 P c$ transcript was observed in swi6 mutant even in nitrogen-rich medium, which is similar to that of wild-type sample under nitrogen starvation conditions (Figure 2, compare lane 3 to lane 2), and is further elevated in the nitrogen minus medium (Figure 2, lane 4). More dramatic results are obtained with mat3Mi transcript. Very low levels of mat3Mi PCR product (estimated size 366 bp) are observed both in nitrogenrich (Figure 2, lane 5) and nitrogen-free medium (Figure 2, lane 6). However, in swi6 strain, while the nitrogen-rich sample shows a barely detectable level of the mat3Mi product (Figure 2, lane 7), nitrogen starvation leads to substantially higher levels of the product (Figure 2, lane 8). Controls for the expression of a normally expressed gene such as DNA pol $\alpha$ showed no change (not shown). Moreover, the specificity of the PCR product as mat $2 P c$ or mat $3 M i$ was confirmed by the absence of any signal when only RNA was used instead of the cDNA, ruling out the templating of the PCR by the genomic DNA that might still be present in the RNA (data not shown). Quantitation by densitometric scanning shows that under nitrogen-rich conditions the mat $2 P c$ transcript is elevated by 7.7-fold in swi6 mutant as compared with the wild-type strain; this level was further elevated by 1.7 -fold in the nitrogen-free medium. Likewise, swi6 mutant shows a 13.8 -fold induction in the level of mat3Mi transcript in nitrogen-free medium as compared with the nitrogen-rich medium. Using the conditions described above, we have been able to successfully detect and quantitate the silent gene transcripts in other silencing mutants as well, even in the homothallic background. The leakiness of the mat $2 P c$ transcript from the silent locus under nitrogenrich conditions is surprising but has been reported earlier (10); it has been speculated that this may result from the partial unfolding of the donor cassette mat $2 P$ during the process of switching in homothallic $\left(h^{90}\right)$ strain (10).

In summary, we have devised a method that allows specific amplification of donor-specific transcripts in the silencing mutants in homothallic 


\section{Benchmarks}

Table 2. Steps Involved in the RT-PCR Protocol

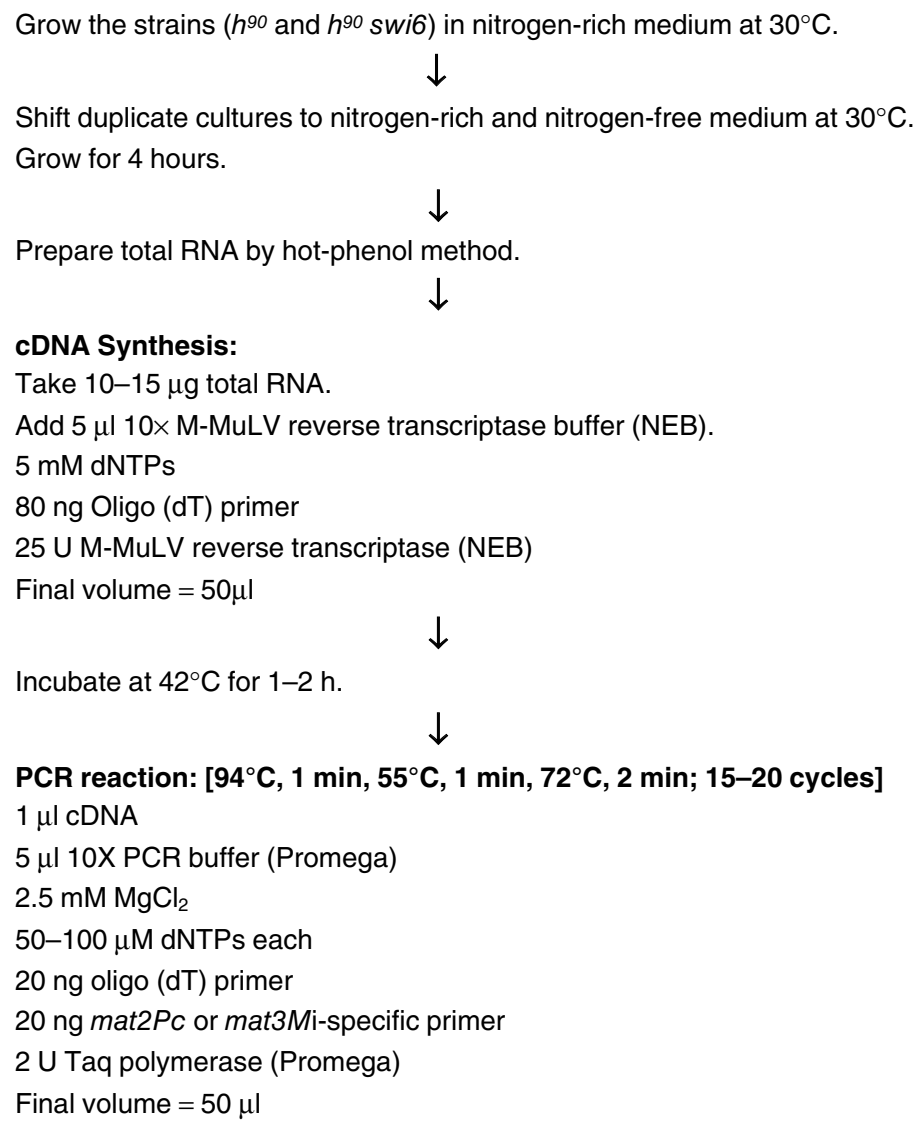

Shift duplicate cultures to nitrogen-rich and nitrogen-free medium at $30^{\circ} \mathrm{C}$. Grow for 4 hours.

Prepare total RNA by hot-phenol method.

\section{$\downarrow$}

cDNA Synthesis:

Take $10-15 \mu \mathrm{g}$ total RNA.

Add $5 \mu \mathrm{l} 10 \times \mathrm{M}-\mathrm{MuLV}$ reverse transcriptase buffer (NEB).

$5 \mathrm{mM}$ dNTPs

$80 \mathrm{ng}$ Oligo (dT) primer

25 U M-MuLV reverse transcriptase (NEB)

Final volume $=50 \mu \mathrm{l}$

Incubate at $42^{\circ} \mathrm{C}$ for $1-2 \mathrm{~h}$.

\section{$\downarrow$}

$\downarrow$

PCR reaction: [ $94^{\circ} \mathrm{C}, 1 \mathrm{~min}, 55^{\circ} \mathrm{C}, 1 \mathrm{~min}, 72^{\circ} \mathrm{C}, 2 \mathrm{~min}$; $15-20$ cycles]

$1 \mu \mathrm{lCDNA}$

$5 \mu \mathrm{l}$ 10X PCR buffer (Promega)

$2.5 \mathrm{mM} \mathrm{MgCl}$

50-100 $\mu \mathrm{M}$ dNTPs each

$20 \mathrm{ng}$ oligo (dT) primer

$20 \mathrm{ng}$ mat2Pc or mat3Mi-specific primer

$2 \cup$ Taq polymerase (Promega)

Final volume $=50 \mu \mathrm{l}$

Resolve the PCR product by agarose gel electrophoresis, followed by Southern blotting and hybridization.

background in $S$. pombe and that allows quantitation of the relative message levels in different mutants. Further multiplexing can facilitate simultaneous detection and quantitation of transcripts from both the donor loci. This approach should be useful for research workers working in the field of gene regulation of the mating type loci in fission yeast.

\section{ACKNOWLEDGMENTS}

This work was supported by Intramural support from the Council of Scientific and Industrial Research, New Delhi, India. We thank Shashi Batra for typing the manuscript and Hemant $K$. Verma for help in drawing the figures. interests.

\section{COMPETING INTERESTS STATEMENT}

The authors declare no competing

\section{REFERENCES}

1. Klar, A.J. 2007. Lessons learned from studies of fission yeast mating-type switching and silencing. Annu. Rev. Genet. 41:213236.

2. Thon, G. and A.J. Klar. 1992. The clrl locus regulates the expression of the cryptic mating-type loci of fission yeast. Genetics 131:287-296.

3. Ekwall, K. and T. Ruusala. 1994. Mutations in rik1, clr2, clr3 and clr4 genes asymmetrically derepress the silent mating-type loci in fission yeast. Genetics 136:53-64.

4. Kelly, M., J. Burke, M. Smith, A. Klar, and D. Beach. 1988. Four mating-type genes control sexual differentiation in the fission yeast. EMBO J. 7:1537-1547.
5. Lorentz, A., L. Heim, and H. Schmidt. 1992. The switching gene swi6 affects recombination and gene expression in the mating-type region of Schizosaccharomyces pombe. Mol. Gen. Genet. 233:436-442.

6. Moreno, S., A. Klar, and P. Nurse. 1991. Molecular genetic analysis of fission yeast Schizosaccharomyces pombe. Methods Enzymol. 194:795-823.

7. Schmitt, M.E., T.A. Brown, and B.L. Trumpower. 1990. A rapid and simple method for preparation of RNA from Saccharomyces cerevisiae. Nucleic Acids Res. 18:3091-3092.

8. Maniatis, T., E.F. Fritsch, and J. Sambrook. 1982. Molecular Cloning: A Laboratory Manual. CSH Laboratory Press, Cold Spring Harbor, NY.

9. Feinberg, A.P. and B. Vogelstein. 1984 A technique for radiolabeling DNA restriction endonuclease fragments to high specific activity. Anal. Biochem. 137:266-267.

10. Singh, J., V. Goel, and A.J.S. Klar. 1998 A novel function of DNA repair gene rhp6 in mating-type silencing by chromatin remodeling in fission yeast. Mol. Cell. Biol. 18:5511-5522.

Received 18 October 2007; accepted 7 November 2007.

S.A.'s present address is Central Drug Research Institute, Chattar Manzil Palace, P.O. Box 173, Lucknow, India.

Address correspondence to Jagmohan Singh, Institute of Microbial Technology, Sector 39A, Chandigarh-160036, India. e-mail:jag@imtech.res.in

To purchase reprints of this article, contact: Reprints@BioTechniques.com 